\title{
Service Supply Chain Network Evolving Model Driven by Service Cooperation Strength
}

\author{
Ma Xuesong \\ Huazhong University of Science and Technology, Wuhan, China \\ ma_xs@163.com
}

\begin{abstract}
This paper discusses the nature of service supply chain network from complex network perspective, and formulates a service supply chain network-evolving model based on service cooperation strength combined with service time and service frequency, which is special characteristics of service supply chain. The analysis result shows the service supply chain network average in-strength and out-strength also follows power law distribution which meets the characteristics of service supply chain.
\end{abstract}

Keywords: Service supply chain; Complex network; Network evolution model

\section{Introduction}

American scholar Ellram, L.M. Et al in 2004 first proposed the concept of service supply chain, then opened the curtain of service supply chain research. Current scholars have different interpretations on the connotation of service supply chain, which can be roughly grouped into three categories: definition from the perspective of service after sales ${ }^{[1]}$; definition from the perspective of outsourcing ${ }^{[2]}$; stressed the distinction between the services and manufacturing ${ }^{[3]}$. S. Alter established three separate service system construction model from the operation of the system, the value chain and life cycle viewpoints, but these three aspects are Mutual fusion and difficult to distinguish, hence there is the practical application certain limitations in actual service system ${ }^{[4]}$.

The current service supply chain definitions are industry-specific and cannot represent the general properties of the service supply chain. The reality of the service supply chain is not only more complex but also dynamic. Previous linear programming method used the classical operational research service supply chain is based on static theory, and assuming the service supply chain hierarchy approach cannot meet current service supply chain complex, dynamic development characteristics.

In recent years the study of complex networks gradually become a hot topic. From a new perspective on network, supply chain system was analyzed to reveal the mechanism of dynamic evolution of service supply chain and made a series of significant research methods and conclusions. How to build a network evolution model to express the unique attributes of the service in line with the supply chain becomes quite significant.

\section{Literature Review}

Thadakamalla et al. first proposed the supply chain network structure model ${ }^{[5]}$. Barrat et al. proposed a BBV model which both edge and node weights evolution are dynamic by phasing into the network weights evolutionary mechanisms ${ }^{[6]}$. But this model does not take directional network into account. Bing-Hong $\mathrm{W}$ proposed a weighted network model driven by traffic flow, assuming that traffic demand and the actual traffic load across the network increase with the network grow, and the growth rate is constant ${ }^{[7]}$. The weight is set to be a basic amount which independent of the connection to drive network evolution. Yang Qin, Yao Juan developed a supply chain network evolution model based on TDE model ${ }^{[8]}$. It obtained the analytical expression of in-out intensity distribution for both nodes and network. But they did not consider the old interconnection between nodes, and the weight was independent of connection which has a certain discrepancy with the actual situation of service supply chain.

In summary, most of the existing supply chain network and evolution models are for the traditional product supply chain, and its weight indicators are not adapted to obtain service supply chain 
characters. Only consider either service time or service frequency is not a more comprehensive description of the unique attributes of the service supply chain.

In reality, the service supply chain is formed by cooperation of each service members. The relations between the various members will vary with the level of cooperation varies. Thus, the evolution weight indicators need to be able to express this service partnership and strength. But most of the existing studies have overlooked this point. In order to fill this study gap, the re-establishment of supply chain evolution model to express the special service characteristics of the supply chain network is studied in this papaer.

\section{Service Supply Chain Network Evolution Model}

Construction of service supply chain model is inseparable from the basic service features. Service cooperation is the key to one of the core features of the service system and the service supply chain ${ }^{[9]}$. Measurement indicator of services cooperation is the service cooperation strength. Service cooperation strength means the service interaction frequency (number of interactions during service transactions) and the length of service duration (service cooperative behavior sustained length of time) between service provider and customer ${ }^{[10]}$.

Based on analysis above, this paper deems the service supply chain network is evolved by service cooperation strength with growth of each node weighted. Therefore, it creates a new index - the service cooperation strength $=$ service time $\times$ service frequency, as a service-weighted index to build the service supply chain evolution model. Either service provider or customer can initiate the service needs and then search the service partner to meet the service demand. Hence service supply chain network is directed weighted network.

Let $G=(N, W)$ as a service supply chain network consists of $N$ member nodes and a set of weighted edge $W$. The matrix element $\boldsymbol{w}_{i j}$ represents the edge weight from service member $i$ to the members $j$, here $\boldsymbol{w}_{i j} \geq 0$, namely service cooperation strength among the node members. It represents the node between the two service members no service relationship when $\boldsymbol{w}_{i j}=0$.

\subsection{Definitions}

Definition 1. The nodes strength between nodes $s_{i}$ in service supply chain network can be depicted as

$$
S_{i}=\sum_{j \in N_{i}} W_{i j}
$$

Goh K I et al.' s study [18] shows that nodes strength and node degree $k i$ has no relevance scale, namely $\mathrm{S} \sim \mathrm{k}^{\beta} . K i$ indicates the degree of node $i$, that is, the number of other nodes connected to the node.

Definition 2. If the limit $\lim _{t \rightarrow \infty} P\{S I(t)=s\}$ exists and $\sum_{s=0}^{\infty} \lim _{t \rightarrow \infty} P\{S I(t)=s\}=1$, then call $\operatorname{limit}_{\lim } P\{S I(t)=s\}$ as a steady-state average in-intensity distribution of the network, denoted $\boldsymbol{P}\{\boldsymbol{S I}=\boldsymbol{s}\}$.

Definition 3. If the limit $\lim _{t \rightarrow \infty} P\{S O(t)=s\}$ exists and $\sum_{s=0}^{\infty} \lim _{t \rightarrow \infty} P\{S O(t)=s\}=1$, then call limit $\lim _{t \rightarrow \infty} \boldsymbol{P}\{\boldsymbol{S O}(t)=s\}$ as a steady-state average out-intensity distribution of the network, denoted $\boldsymbol{P}\{\boldsymbol{S O}=\boldsymbol{s}\}$.

\subsection{Service Supply Chain Network Evolving Model}

Based on the above description of characteristics and definitions, introduced the topological growth and strength coupling synchronization mechanism, combined with service supply chain nodes arrival process follows the Poisson distribution flow, the evolutionary models of service supply chain complex networks can be established as follows.

(1) Initial. Initial service supply chain have service member nodes $\boldsymbol{m}_{\boldsymbol{b}}$, the initial weight of each edge is $\boldsymbol{w}_{\boldsymbol{0}}=\mathbf{1}$, constitutes BA network, that is, without repeating edge and self-connecting edge.

(2) Growth. At each time step t, the new nodes are added to the initial network according to Poisson distribution, each new node with $m$ edges are connected to the $m$ different nodes existed in networks 
already $\left(\boldsymbol{m} \leq \boldsymbol{m}_{\boldsymbol{0}}\right)$. The in-edge number of new nodes obey the binomial distribution $\boldsymbol{B}(\boldsymbol{m}, \boldsymbol{p})$, the out-edge number of new nodes obey the binomial distribution $\boldsymbol{B}(\boldsymbol{m}, \mathbf{1}-\boldsymbol{p}), \boldsymbol{p} \in(\boldsymbol{0}, \mathbf{1})$.

(3) Preferential connection. New node connects to existed node according to node strength. The node with bigger strength connects to new node preferentially. Let $V$ be the set of adjacent node $i$, then:

(1) When select an existing node $i$ as the end point, the probability $\Pi_{1}$ of selecting $i$ depends on the in-strength of $i$.

$$
\Pi_{1}=S I_{i} / \sum_{j \in V} S I_{j}
$$

(2) When select an existing node $i$ as the strat point, the probability $\Pi_{2}$ of selecting $i$ depends on the out-strength of $i$.

$$
\Pi_{2}=S O_{1} / \sum_{j \in V} S_{j}
$$

(4) Node exit. When a new node joins the network, with probability $q$ randomly delete a node in the network, meanwhile will remove all edges connected to the node. At time $t$, the number of average exit nodes is $q \lambda \boldsymbol{t}$.

(5) Weight evolution. New nodes and node exiting will bring dynamic changes in weight.

(1) When a new node is added, that new customer requirements for service or when new service providers to provide customers with added services, network connecting all the possible edge weight according to the following intensity coupling dynamics mechanism to be updated.

$$
\boldsymbol{w}_{i j}= \begin{cases}\boldsymbol{w}_{i j}+\boldsymbol{1}, & \text { By probability } \boldsymbol{W} \boldsymbol{p}_{i j} \\ \boldsymbol{w}_{i j} & \text { By probability } \boldsymbol{l}-\boldsymbol{W} \boldsymbol{p}_{i j}\end{cases}
$$

Where $\mathrm{W}$ is total increased amount of edge weight, reflecting a growth rate of total service supply chain network services cooperative strength. Continued growth in demand for services reflected in the growth of aggregate service cooperation strength, play a driving force role in the evolution of the network.

The service time of nodes obey the negative exponential distribution of probability density function $\mu e^{-\mu t}, t \geq 0$, then the network growth rate of total service cooperation strength is $W=\mu e^{-\mu t} T_{i j}, T_{i j}$ indicates the service frequency of nodes. According to literature, service frequency is evolved as following equation. Without loss of generality, let $w_{i j}=T_{i j}$. $T_{\mathrm{ij}}(t+1)=T_{i j}(t)+1$. Assume that the network node is added with the constant growth of the service time, then the edge weight is evolved as following formula. $W_{\mathrm{ij}}(t+1)=T_{i j}(t+1) \mu e^{-\mu t}$.

Taking into account the evolution had little correlation with the initial state of the network, limited

$$
\boldsymbol{W} \boldsymbol{p}_{i j} \in[0,1] . \boldsymbol{p}_{i j}=\boldsymbol{w}_{i j} / \sum_{a, b} \boldsymbol{w}_{a b}
$$

(2) When a node exit, network traffic will change accordingly, the edge weight is updated by the following mechanism.

$$
\Delta w_{j i}=S I_{v} \frac{w_{j i}}{\sum_{a, b} w_{a b}}, \Delta w_{i j}=S O_{v} \frac{w_{i j}}{\sum_{a, b} w_{a b}},
$$

$\Delta \boldsymbol{w}_{j i}$ indicates in-edge flow change amount of node $i, \Delta \boldsymbol{w}_{i j}$ indicates out-edge flow change amount of node $i$.

\subsection{Resolution Process}

The arrival process of customer nodes obey Poisson distribution with parameter $\lambda$. Set $a(t)$ as the total number of nodes at time $t$ to join the service supply chain network, then $E(a(t))=\lambda t$, represents averagely there are $\lambda t$ nodes to join the network at time $t$. Set $d(t)$ as the total number of nodes at time $t$ to exit the service supply chain network after service finish, then $E(d(t))=q \lambda t$, represents averagely there are $q \lambda t$ nodes to exit the network at time $t$. 
Thus, at time $t$ the total number of nodes in service supply chain network $N(t)$ can be expressed as $E(N(t))=m_{0}+\lambda t-q \lambda t$, When $t$ is large enough, $m_{0}$ is negligible, then $E(N(t))=\lambda t-q \lambda t=(1-q) \lambda t$. When a new node joins the network, the weights change rate of existed edge in network is

$$
\begin{aligned}
& \frac{d w_{j i}(t)}{d t}=\left[\left(w_{j i}+1\right) W p_{j i}+w_{j i}\left(1-W p_{j i}\right)-w_{j i}\right] \lambda \\
& =\lambda W p_{j i}=\lambda W w_{j i} / \sum_{a, b} w_{a b}=\lambda T_{i j} \mu e^{-\mu x} w_{j i} / \sum_{a, b} w_{a b}
\end{aligned}
$$

In-strength of network at time $t$ is $S \boldsymbol{I}_{i}(\boldsymbol{t})=\frac{\boldsymbol{S I}_{\boldsymbol{i}}\left(\boldsymbol{t}_{i}\right)}{\boldsymbol{t}_{i}^{\mathrm{A}}} \boldsymbol{t}^{\mathrm{A}}, \quad A=\frac{(1-p) m+\mu e^{-\mu t} T_{i j}}{m+\mu e^{-\mu} T_{i j}}$

Out-strength of network at time $t$ is $\boldsymbol{S O}_{\boldsymbol{i}}(\boldsymbol{t})=\frac{\boldsymbol{S O}_{\boldsymbol{i}}\left(\boldsymbol{t}_{i}\right)}{\boldsymbol{t}_{i} \boldsymbol{t}^{B}}, \quad B=\frac{p m+\mu e^{-\mu t} T_{i j}}{m+\mu \boldsymbol{e}^{-\mu t} T_{i j}}$

Given the steady state in-strength and out-strength of node in Poisson arrival process does not exist, but the average steady-state in-strength and out-strength of network exists ${ }^{[15]}$. Therefore, this paper only analyzes average steady-state in-strength and out-strength of network as follows.

Average steady-state in-strength of network $P(S I=s)$ is

$$
\begin{aligned}
& P(S I=s)=\lim _{t \rightarrow \infty} P(S I(t)=s) \\
& \approx \lim _{i \rightarrow \infty}\left(\frac{1}{(\lambda-q \lambda) t} \sum_{i=1}^{\infty}\left(\frac{\lambda t}{A}\right)\left(\frac{1}{s}\right)^{1+1 / 4} \cdot \sum_{l=0}^{m}\left[C_{m}^{t} p^{l}(1-p)^{m-l} \cdot \frac{\exp \left[-\lambda\left(\frac{l}{s}\right)^{1 / 4} t\right]\left[\lambda\left(\frac{l}{s}\right)^{1 / 4} t\right]^{t^{i-1}} \cdot l^{1 / 4}}{(i-1) !}\right]\right\} \text { When } t \rightarrow \infty, A=\frac{(1-p) m+\mu e^{-\mu t} T_{i j}}{m+\mu e^{-\mu t} T_{i j}}=1-p \\
& =\lim _{l \rightarrow \infty}\left\{\frac{1}{(1-q) A s^{1+1 / 4}} \sum_{l=0}^{m} C_{m}^{l} p^{l}(1-p)^{m-t} l^{1 / A} \cdot \exp \left[-\lambda\left(\frac{l}{s}\right)^{1 / 4} t\right] \sum_{i=1}^{\infty} \frac{\left[\lambda\left(\frac{l}{s}\right)^{1 / 4} t\right]^{i-1}}{(i-1) !}\right\} \\
& =\frac{1}{(1-q) A S^{1+1 / /}} \sum_{l=0}^{m}\left[C_{m}^{l} p^{l}(1-p)^{m-l} l^{1 / 4}\right]
\end{aligned}
$$

Thus $P(S I=s)=\frac{1}{(1-q) A s^{1+1 /(1-p)}} \sum_{l=0}^{m}\left[C_{m}^{l} p^{l}(1-p)^{m-l} l^{1 /(1-p)}\right]$

Average steady-state out-strength of network $P(S O=S)$ is

$$
\begin{aligned}
& \begin{array}{l}
P(S O=s)=\lim _{i \rightarrow \infty} P(S O(t)=s) \\
\left.\approx \lim _{l \rightarrow \infty} \frac{1}{(\lambda-q \lambda) t} \sum_{i=1}^{\infty}\left(\frac{\lambda t}{B}\right)\left(\frac{1}{s}\right)^{1+1 / \beta} \cdot \sum_{l=0}^{m}\left[C_{m}^{l}(1-p)^{l} p^{m-l} \cdot \frac{\exp \left[-\lambda\left(\frac{l}{s}\right)^{1 / B} t\right]\left[\lambda\left(\frac{l}{s}\right)^{1 / B} t\right]^{i-1} \cdot l^{1 / B}}{(i-1) !}\right]\right\} \text { When } t \rightarrow \infty, B=\frac{p m+\mu e^{-\mu t} T_{i j}}{m+\mu e^{-\mu t} T_{i j}}=p
\end{array} \\
& =\lim _{t \rightarrow \infty}\left\{\frac{1}{(1-q) B S^{l+1 / B}} \sum_{l=0}^{m} C_{m}^{l}(1-p)^{l} p^{m-l} l^{1 / B} \cdot \exp \left[-\lambda\left(\frac{l}{s}\right)^{1 / B} t\right] \sum_{i=1}^{\infty} \frac{\left[\lambda\left(\frac{l}{s}\right)^{1 / B} t\right]^{i-1}}{(i-1) !}\right\} \\
& =\frac{1}{(1-q) B s^{1+1 / \beta}} \sum_{l=0}^{m}\left[C_{m}^{l}(1-p)^{l} p^{m-l} l^{1 / B}\right]
\end{aligned}
$$

Thus $P(S O=s)=\frac{1}{(1-q) B s^{1+1 / p}} \sum_{l=0}^{m}\left[C_{m}^{l}(1-p)^{l} p^{m-l} l^{1 / p}\right]$

It can be known that the average steady state in-strength and out-strength of the network follow Power law distribution respectively.

$$
P(S I=s) \sim s^{-\alpha}, \alpha=1+\frac{1}{1-p} ; P(S O=s) \sim s^{-\beta}, \beta=1+\frac{1}{p}
$$

Since $\mathrm{p} \in(0,1)$, it can be seen the power-law exponent $\alpha$ and $\beta$ are in $(2, \infty)$ range. This conclusion is same as power law distribution range of literature ${ }^{[11]}$ and proved its validity. 


\section{Conclusions}

This article establishes a directed weighted general evolutionary model of service supply chain complex network, by combining complex network evolution ideology of flow-driven and connected time-driven together, creating a new weight indicator-service cooperation strength to characterize the weighted intensity of nodes in service supply chain complex network. This model can describe the unique attributes of the service supply chain complex network and much more accord with the actual service supply chain generality. It improves the limitation of single weight indicators in existing complex network evolution models.

As can be seen from the model analysis, both average steady-state in-strength and out-strength of the service supply chain complex network meet the power law distribution. This result fits the features of most of complex network with cooperation relationship and in line with specific characters of customer impact and collaborative service supply chain, which verified the validity of this model.

The model does not consider the waiting time of new nodes, service provider' $\mathrm{s}$ vacations and other special services system cases. And customer arrival process also follows other distributions besides Poisson process in real supply chain network, these more general characterization remains to be further studied.

\section{References}

[1] Ellram, L. M., W. L. Tate, C. Billington. Understanding and managing the service supply chain. [J]. Supply Chain Management. 2004, 40(4) 17-32.

[2] Dirk de Waart, Steve Kemper. Five Steps to Service Supply Chain Excellence. [J]. Supply Chain Management Review. 2004, 8(1), 28-35

[3] Li-yin J I N. Service Supply Chain Management, Customer Satisfaction and Business Performance[J]. Chinese Journal of Management Science, 2006, 2: 100-106.

[4] S. Alter. Service system fundamentals: Work system, value chain, and life cycle. [J]. IBM Syestem Journal. 2008, Vol 47, No1.

[5] THADAKAMALLA H P,RAGHAVAN U N, KUMARA S, et al. Survivability of multiagent-based supply networks:A topological perspective [J].IEEE Intelligent Systems,2004,19(5):24-31

[6] Barrat A, Barthélemy M, Vespignani A. Modeling the Evolution of Weighted Networks[J]. Physical Review E, 2004, 70(6): 1-13.

[7] Bing-Hong W, Tao W W X Z. A weighted complex network model driven by traffic flow [J]. Physics, 2006,4: 012.

[8] Yang Qin, Yao Juan. Weighted directed supply chain network based on TDE model [J]. Journal of Systems \& Management, 2013, 22(5)

[9] Ennew C T, Binks M R. Impact of participative service relationship on quality, satisfaction and retention: An exploratory study [J]. Journal of Business Research, 1999, 46(2): 121-132.

[10] Li Huifeng. Research on Contracting for Service Co-Production [D]. Dalian University of Technology,2013.

[11] Jin-Li G. The bilateral power-law distribution model of supply chain networks [J]. Acta Physica Sinica, 2006, 55(8)3916-06. 